\title{
A General Technical Route for Parameter Optimization of Ship Motion Controller Based on Artificial Bee Colony Algorithm
}

\author{
Yanfei Tian, Liwen Huang, and Yong Xiong
}

\begin{abstract}
The most practical application in industrial process control is still the conventional PID control algorithm. However, PID parameter tuning and optimizing, where the difficulty lies in as well, is an important issue in the field of automatic control. It is the direction to achieve global optimization of PID parameters using intelligent optimization algorithm. Aiming at the difficult problems in parameter tuning of PID controllers in industrial control, A parameter tuning and optimization method based on artificial bee colony algorithm is proposed, the parameter of PID controller need to be tuned is seen as the nectar source, the high-quality combination of parameters is searched using the unique role change mechanism of the bees; and the ITAE index is selected as the objective function for parameter optimization. Simulation implemented on MATLAB-Simulink proved the proposed process was of feasibility and availability in the optimization work. The process and results showed that proposed technical route was simple and feasible with strong adaptability, making it an effective computer aided offline parameter optimization method for PID controller.
\end{abstract}

Index Terms - Ship engineering, automation, PID controller, intelligent parameter optimization, artificial bee colony algorithm.

\section{INTRODUCTION}

At present, the industrial automation level has become an important symbol to measure the level of modernization in all walks of life. At the same time, it also reflects a country's development speed at some point. Also the development of the control theory has experienced three stages characterized by the classical control theory, modern control theory and intelligent control theory [1].

As control theories rapidly develop, many advanced control algorithms now have appeared. But so far, the most practical application in industrial process control is still the conventional PID control algorithm. This is because the PID control has a simple structure and is easy to be implemented with perfect performance and strong robustness. What is more, principle of PID algorithm is concise, with clear

Manuscript received December 19, 2015; revised February 19, 2016. This work was supported by: (1) the Fundamental Research Funds for the Central Universities (specifically supported by the self-determined and innovative research funds of WUT, Grant No. 2014-JL-010), (2) the Natural Science Foundation of Hubei Province (Grant No. 2014ZFB878), and (3) the Key Project in the National Science \& Technology Pillar Program (Grant No.2015BAG20B05)

Yanfei Tian, Liwen Huang, and Yong Xiong are with the School of Navigation, Wuhan University of Technology (WUT), China, and the Hubei Key Laboratory of Inland Shipping Technology, WUT, China (e-mail: feifei_whut@163.com, 1whuang@whut.edu.cn,bear_brave@163.com). physics meanings of the parameters and a complete theoretical system, which the majority of control engineers are familiar with [2].

However, PID parameter tuning and optimizing, where the difficulty lies in as well, is an important issue in automatic control fields. The conventional PID algorithm especially requires its parameters high-integrated and often fails to achieve satisfied control results. Due to a poor parameter setting at the application scene of PID theory or controller, resulting in unsatisfied control effect, people over the years have dedicated to the study parameter setting method and a variety of the methods have been put forward.

There are plenty of methods for PID parameter tuning, for example, the classical Z-N method, simplex method and some intelligent algorithms such as Artificial Neural Network (ANN), Genetic Algorithm (GA) [3], etc. The classification of those kinds of methods can be seen in [2]. In case of the low efficiency and some unsatisfactory, it is the direction to achieve global optimization of PID parameters using intelligent optimization algorithm [4].

PID controller is widely used in practice for ship course control. However, the large time delay, large inertia and nonlinearity of ship motion, make the selection of PID parameters more difficult [5]. Aiming at the difficult problems in parameter tuning of PID controllers in industrial control, we will propose a general technical route for parameter optimization of ship motion PID controller based on Artificial Bee Colony (ABC) algorithm, where the parameters of PID controller need to be tuned and optimized will be treated as the nectar source, high-quality combination of parameters will be searched using the unique role change mechanism of the bees, and the integral of time multiplied by the absolute value of error (ITAE) will be selected as the objective function for parameter optimization. The proposed general technical route will be tested via MATLAB-Simulink based simulations.

\section{The Artificial Bee Colony (ABC) Algorithm}

\section{A. Glance at ABC Algorithm}

Introduced by D. Karaboga [6]-[9], the ABC algorithm is a nature-inspired algorithm based on the intelligent foraging behavior of honey bee swarm and has been used to find an optimal solution in numeric optimization problems [10]. Based on many benchmark functions, researches [7]-[9] showed the $\mathrm{ABC}$ algorithm was competitive to other population-based algorithms, such as GA, Particle swarm optimization (PSO), Differential Evolution (DE), evolution 
strategies and Particle Swarm inspired Evolutionary Algorithm (PS-EA), etc., with an advantage of employing fewer control parameters [11].

Since its invention in 2005, ABC algorithm has attracted a lot of attention and been applied to solve many kinds of problem beside numerical function optimization [10]. A survey about applications of $\mathrm{ABC}$ algorithm is provided by Karaboga et al. in [12]. Besides, there are now many types (or improved versions) of the original $\mathrm{ABC}$ due to a large number of researchers' initiative interests in it and (or) making it more suitable for various problems. To differentiate, we call the algorithm proposed in 2005 the standard ABC, what is the one the paper here refers to.

\section{B. Basic Principle Description of ABC Algorithm}

Accordingly [6]-[10], colony of artificial bees consists of three groups of bees to search foods generally, which includes employed bees, onlookers and scouts. The first half of colony consists of the employed artificial bees and the second half includes the onlookers. The employed bees search the food around the food source in their memory. They perform waggle dance upon returning to the hive to pass their food information to the other of the colony (the onlookers). The onlookers are waiting around the dance floor to choose any of the employed bees to follow based on the nectar amount information shared by the employed bees. The employed bee whose food source has been exhausted by the bees becomes a scout who must find new food source. For every food source, there is only one employed bee. In other words, the number of employed bees is equal to the number of food sources around the hive. In ABC algorithm, the position of a food source represents a possible solution to the optimization problem and the nectar amount of a food source corresponds to the quality of the associated solution (fitness value).

\section{Implement Steps of ABC Algorithm}

Briefly and accordingly [6]-[10], the main steps of implementing the $\mathrm{ABC}$ algorithm are given below.

Step 1: Randomly generate the initial population of $\mathrm{N}$ food sources within the range restricted by boundaries of the variables according to Eq. (1).

$$
x_{i}^{j}=x_{\min }^{j}+\operatorname{rand}[0,1]\left(x_{\max }^{j}-x_{\min }^{j}\right)
$$

where $i=1 \ldots N, j=1 \ldots D . N$ is the number of food source and $D$ is the number of variables to be optimized.

Step 2: Evaluate each food source by calculating its fitness (i.e. calculate the nectar amount) according to Eq. (2).

$$
\text { fit }_{i}=\left\{\begin{array}{lll}
1 /\left(1+f_{i}\right) & \text { if } & f_{i} \geq 0 \\
1+\operatorname{abs}\left(f_{i}\right) & \text { if } & f_{i}<0
\end{array}\right.
$$

where $f_{i}$ is the cost value of solution $x_{i}$. For maximization problems, the cost function can be directly used as a fitness function.

Step 3: Each employed bee searches a candidate food source $v_{i}$ according to Eq. (3). Evaluate the candidate food source and apply greedy selection to select a better one as the new food source.

$$
v_{i j}=x_{i j}+\phi_{i j}\left(x_{i j}-x_{k j}\right)
$$

where $j$ is a random integer within $[1, D]$ and $k \in\{1,2, \cdots N\}$ is a randomly chosen index that is different from $i . \phi_{i j}$ is a uniformly distributed real random number within $[-1,1]$.

Step 4: Calculate probability based on fitness of the solutions in the population. Each onlooker selects a food source according to Eq. (4) by roulette wheel selection and generates a candidate solution according to Eq. (3).

$$
p_{i}=\frac{f i t_{i}}{\sum_{n=1}^{S N} f i t_{n}}
$$

Step 5: Evaluate the candidate food source and select a better one as the new food source according to greedy selection.

Step 6: Memorize the best food source position (solution) found so far.

Step 7: If the position of a particular food source cannot be improved through the predetermined number of trials "limit", then select it as an abandoned one. Replace the solutions by a new position that is randomly produced by a scout according to Eq. (1).

Step 8: Repeat the procedure from step 3 until the termination criterion is met. When the algorithm is terminated, the position of optimal food source and its nectar amount are the optimum values of the decision variables and objective function for the considered problem.

\section{PARAMETER OPTIMIZATION OF SHIP PID CONTROLLER}

\section{A. The PID Control System of Ship Course}

The ship course PID control system drawn in MATLAB-Simulink library is shown in Fig. 1.

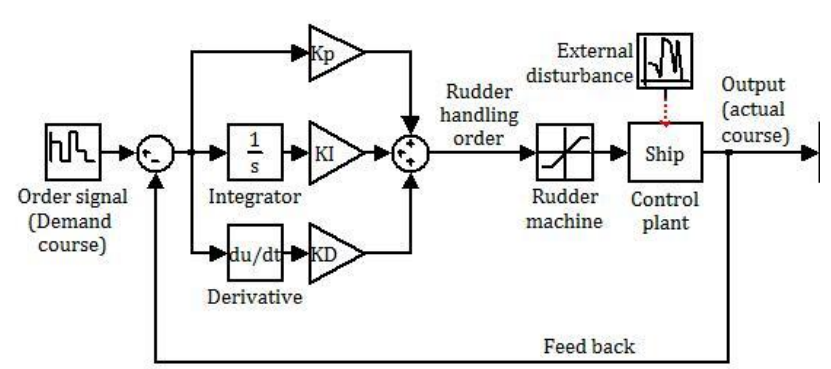

Fig. 1. A PID course control system of ship course.

The algorithm of PID controller was represent by Eq. (5).

$$
\delta(t)=K_{\mathrm{P}} e(t)+K_{\mathrm{I}} \int_{0}^{t} e(t) \mathrm{d} t+K_{\mathrm{D}} \frac{\mathrm{d} e(t)}{\mathrm{d} t}
$$

And, $e(t)$ means the tracking error calculated by Eq. (6).

$$
e(t)=y_{r}(t)-y(t)
$$

Additionally, $y_{r}(t)$ represents the command signal for 
reference, while $y(t)$ represents the actual signal feedback.

\section{B. Problem Description of Parameter Optimization for the Control System}

The problem of PID parameter optimization for ship course control can be briefly described in a sentence that a set of suitable KP, KI, KD need to be determined to make a certain or more evaluation indexes perform best.

There are several most widely used error integral performance indexes (criteria) to evaluate a control system, such as integral of absolute value of error (IAE), integral of squared error (ISE), integral of time multiplied by the absolute value of error (ITAE), integral of time multiplied by squared error (ISTE). There is no doubt that such indexes can be utilized as the objective functions. In view of this, an objective function can be chosen form the following equations (7) to (10).

$$
\begin{gathered}
J_{\mathrm{IAE}}=\int_{0}^{\infty}|e(t)| \mathrm{d} t \\
J_{\mathrm{ISE}}=\int_{0}^{\infty}\left|e^{2}(t)\right| \mathrm{d} t \\
J_{\mathrm{ITAE}}=\int_{0}^{\infty} t \times|e(t)| \mathrm{d} t \\
J_{\mathrm{ISTE}}=\int_{0}^{\infty} t \times\left|e^{2}(t)\right| \mathrm{d} t
\end{gathered}
$$

where the nonlinear $J$ is the objective function corresponding to different error integral performance indexes, and $e(t)$ is calculated by Eq. (6).

\section{A Search for Best Solutions Based on ABC Algorithm}

By analysis, we believed that existed the following correspondence among actual act of honey bees, solving of optimization problems based on ABC algorithm and parameter optimization of ship course PID controller, which was listed in Table I.

\begin{tabular}{|c|c|c|}
\hline $\begin{array}{l}\text { Actual act of } \\
\text { honey bees }\end{array}$ & $\begin{array}{l}\text { Solving of optimization } \\
\text { problems based on ABC } \\
\text { algorithm }\end{array}$ & $\begin{array}{l}\text { Parameter optimization of } \\
\text { ship course PID controller }\end{array}$ \\
\hline $\begin{array}{l}\text { Nectar source } \\
\text { location }\end{array}$ & Feasible solutions & $\begin{array}{l}\text { Various values of the } \\
\text { parameters KP,KI,KD }\end{array}$ \\
\hline $\begin{array}{l}\text { Nuctar source } \\
\text { earning degree }\end{array}$ & $\begin{array}{l}\text { Quality of the feasible } \\
\text { solutions }\end{array}$ & $\begin{array}{l}\text { Performance index value in } \\
\text { accordance with different } \\
\mathrm{KP}, \mathrm{KI}, \mathrm{KD}\end{array}$ \\
\hline $\begin{array}{l}\text { The speed of } \\
\text { the foraging }\end{array}$ & $\begin{array}{l}\text { Speed of optimizing } \\
\text { fessible solutions }\end{array}$ & $\begin{array}{l}\text { Speed of optimizing the } \\
\text { performance index }\end{array}$ \\
\hline $\begin{array}{l}\text { The maximum } \\
\text { earning degree }\end{array}$ & The optimal solution & $\begin{array}{l}\text { The optimal KP, KI, KD } \\
\text { minimizing the } \\
\text { performance index }\end{array}$ \\
\hline
\end{tabular}

TABLE I: CORRESPONDENCE AMONG ACTUAL ACT OF HONEY BEES, SOLVING OF OPTIMIZATION PROBLEMS BASED ON ABC ALGORITHM AND PARAMETER OPTIMIZATION OF SHIP COURSE PID CONTROLLER

Thus it can be inferenced that the PID controller parameter optimization problem was able to be inverted as a nonlinear function optimization problem, and then could be solved using artificial bee colony algorithm. The main steps to solve the problem were as follows:

1) Determining control variables (parameters to be optimized) and constructing an objective function.

2) Initializing algorithm settings.

3) Aiming at optimizing the objective function, executing the artificial bee colony algorithm.

4) Outputting parameter optimization results (the optimal parameters).

5) Applying the optimal parameters and analyzing the control effect.

\section{Simulation of the Model and CAlCUlating Flow}

\section{A. A SIMULINK Model Combine Control System and Performance Calculation}

The SIMULINK model simulating step response and calculating error performance of the system is shown in Fig. 2.

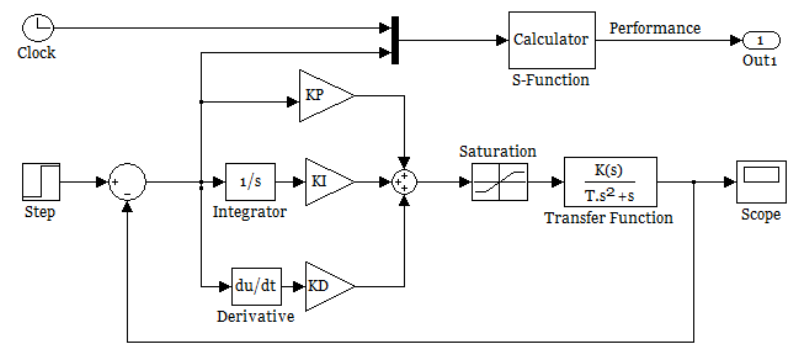

Fig. 2. Simulink model of step signal based course control system (the lower half) and error performance calculation (the upper half) .

where:

1) The concrete content of TF block calculating ship motion was given by converting the following differential equation (11) according to [13], [14].

$$
T \ddot{\psi}+\dot{\psi}=K \delta
$$

Taking YULONG as an example, the ship's maneuvering character indices were: $T=216.58$ and $K=0.48$ [13].

2) The S-function block (Calculator) was used to calculate error performance according to different performance requirements.

Selection of objective function should be combined with the characteristics of the problem to be solved, and be convenient for application. ITAE is one of the best performance indexes applied to evaluate a single input and single output (SISO) control system and the adaptive control system. And ITAE is always chosen as the objective function to be optimized a conventional PID control system to obtain better control effect. Here we chose ITAE as the index for investigation, thus the main and concrete content of the Calculator block was programmed according to Eq. (9).

\section{B. Settings of the ABC Algorithm}

Meanings of the variables and their values used in the program are listed below.

Population size: $\mathrm{NP}=30$.

Number of foods: FoodNumber=NP/2.

Number of the scouters: SearchNumber $=5$.

Maximum cycle: $\operatorname{maxCycle}=100$.

Tries Limit: limit $=5$.

Upper bound of [KP KI KD]: Ub=[300 300 300]. 
Lower bound of [KP KI KD]: $\mathrm{Lb}=\left[\begin{array}{lll}0 & 0 & 0\end{array}\right]$.

\section{Parameter Optimization Results based in ABC Algorithm}

Optimization processes of controller variables (specifically $K_{P}, K_{I}, K_{D}$ ) can be seen in Fig. 3 and the final optimal values are: $K_{P}=159, K_{I}=0$ and $K_{D}=300$.
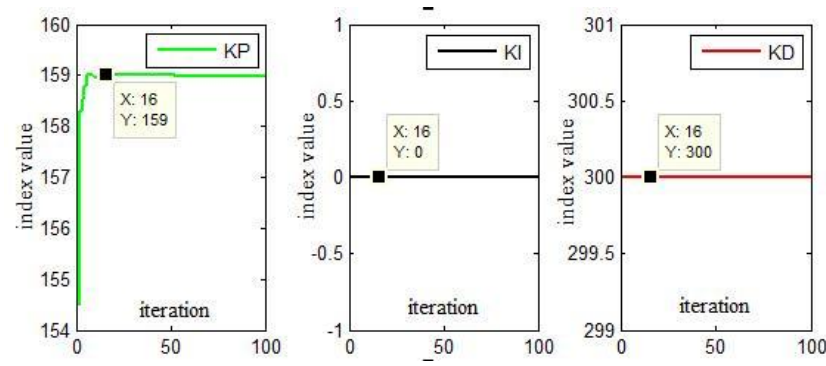

Fig. 3. Optimization processes of the control variables (PID parameters).

For comparison, we introduce a set of un-optimized parameters (e.g. $K_{P}=260, K_{I}=10, K_{D}=210$ ). And then, system step response corresponding with the optimal and un-optimized PID parameters are separately shown in Fig. 4 and Fig. 5. Changes of the system performance index corresponding with the optimal and some un-optimized PID parameters are shown in Fig. 6 and Fig. 7.

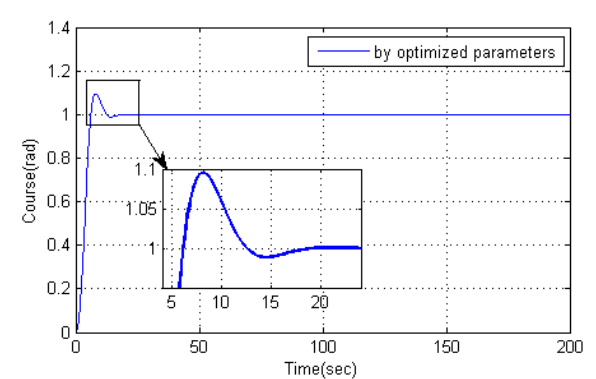

Fig. 4. System step response corresponding with optimal PID parameters.

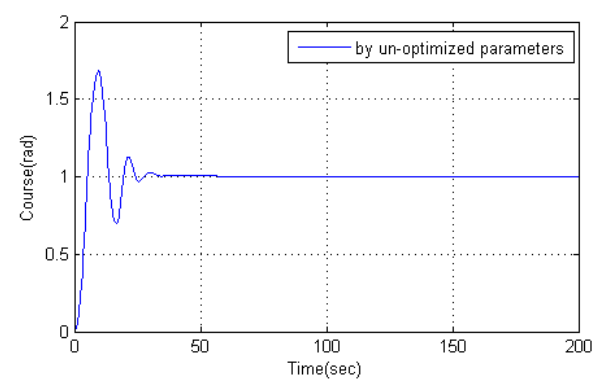

Fig. 5. System step response corresponding with un-optimized parameters.

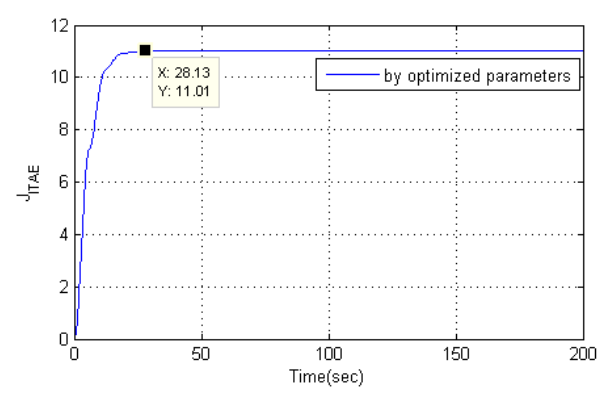

Fig. 6. System performance corresponding with the optimal parameters.

It can be seen that the ABC algorithm was applicable to solve the parameter optimization problem, the given the process with the MATLAB-SIMULINK based simulation model was workable. Compared with the results provided by un-optimized parameters, simulation showed that the optimal parameters produced: (1) high tracking speed (the time from initial moment to steady state less than 10 seconds); (2) only one small overshoot (less than 10\%, shown in Fig. 4) or undesired, redundant oscillation elements; and (3) a best performance $\left(\min J_{\mathrm{ITAE}}=11.01\right)$.

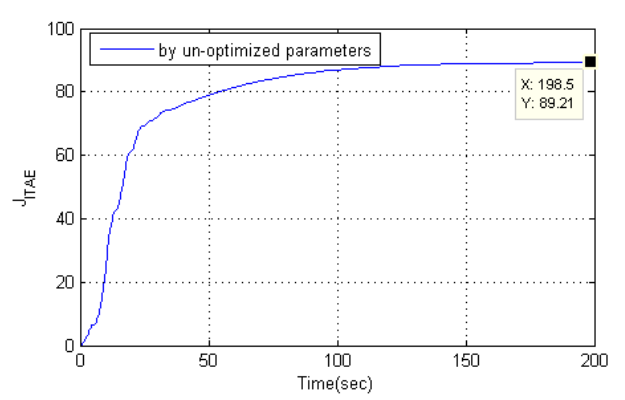

Fig. 7. System performance corresponding with un-optimized parameters.

Generally, it can be summarized that the ABC algorithm was applicable to solve the problem when applied for parameter optimization of ship motion PID controller and the optimized parameters produced a better effect, reaching the goal of a rapid, accurate and stable control effect, and minimizing the performance index value.

\section{CONCLUSION AND OUTLOOK}

\section{A. Conclusions}

Parameter tuning is an essential part of kinds of controllers, a global optimization of the parameters can be realized by applying optimization algorithms, meanwhile overcoming the defects of traditional methods. Focusing on such field, this paper have launched the following work:

1) Analyzed and pointed out that achieving global optimization of PID parameters using intelligent optimization algorithm was the development direction of PID controller parameter tuning and optimization.

2) Based on the performance requirements of the control system, analyzed the adaptability between PID parameters optimization problem and $\mathrm{ABC}$ algorithm. By specific describing the ABC algorithm based steps on how to optimize PID parameters for ship course control system, the paper introduced simultaneously a scheme of optimizing the parameters based on $\mathrm{ABC}$ algorithm as well as a MATLAB-SIMULINK based simulation model.

3) Simulation implemented on MATLAB-Simulink proved the proposed process was of feasibility and availability in the optimization work.

4) The process and results showed that proposed technical route was simple and feasible with strong adaptability, making it an effective computer aided offline parameter optimization method for PID controller.

Due to some details, there existed some small overshoot in the system. But it does not affect the feasibility of the 
proposed scheme. Additionally, although a practical control system is far more complicated than the above given example, but the optimization principle of a number of parameters is the same or similar. Thus it can be speculated that the developed scheme can be referenced for parameters optimization of not only a PID but also the other kinds of controllers.

\section{B. Outlooks}

In the follow-up studies, we will focus on:

1) Further study on principles and improvement of $A B C$ algorithm to improve its performance, such as the convergence speed as well as the search precision.

2) Deeper analysis of the control system itself and improvement the objective function, in order to make the optimization goal meet the dynamic requirements of performance indicators as well as Enhance dynamic performance of the control system.

3) Expanding application scope of the proposed technical route for parameter optimization based on $A B C$ Algorithm, and optimizing other kinds of controllers applied in practice, to conform to the actual demands.

\section{ACKNOWLEDGMENT}

Special thanks are given to the research projects for their infrastructural and financial support. Sincere appreciates are delivered to scholars of the references for their outstanding contributions.

\section{REFERENCES}

[1] T. Yang and Y. Shan, "Simulation of PID controller parameter tuning," Urban Construction Theory Research (Electronic Version), vol. 5, no. 16, June 2015

[2] Z. Liu, X. Jiang, and D. Li, "Overview of the tuning methods of the pid controller parameters," Automation of Electric Power Systems, vol. 2, no. 8, pp. 79-83, 1997.

[3] C. Cai and W. Zhou, "Self-tuning PID parameters by using artificial bee colony algorithm," Process Automation Instrumentation, vol. 36 no. 8, pp. 74-77, August 2015.

[4] B. Guo, L. Zou, and X. Qian, "The optimization of PID tuning based on model simulation technology," Manufacture Automation, vol. 37, no. 8 pp. 22-24, August 2015.

[5] Y.ZHU, "Parameters optimization of ship course PID control," Dalian Maritime University, 2012.

[6] D. Karaboga, "An idea based on honey bee swarm for numerical optimization," Technical Report (TR06), Computer Engineering Department, Erciyes University, Kayseri, Turkey, 2005.

[7] D. Karaboga and B. Basturk, "A powerful and efficient algorithm for numerical function optimization: Artificial bee colony (ABC) algorithm," Journal of Global Optimization, vol. 39, no. 3, pp. 459-471, 2007.

[8] D. Karaboga and B. Basturk, "On the performance of artificial bee colony (ABC) algorithm," Applied Soft Computing, vol. 8, no. 1, pp. 687-697, 2008
[9] D. Karaboga and B. Akay, "A comparative study of artificial bee colony algorithm," Applied Mathematics and Computation, vol. 214, no. 1, pp.108-132, 2009.

[10] P. Guo, W. Cheng, and J. Liang, "Global artificial bee colony search algorithm for numerical function optimization," in Proc. 2011 Seventh International Conference on Natural Computation, vol. 3, pp. 1280-1283, July, 2011.

[11] W. Chen, "An artificial bee colony algorithm for uncertain portfolio selection," The Scientific World Journal, vol. 2014, pp. 1-12, 2014.

[12] D. Karaboga, B. Gorkemli, C. Ozturk et al., "A comprehensive survey: Artificial bee colony (ABC) algorithm and applications," Artificial Intelligence Review, vol. 42, pp. 21-57, 2014.

[13] X. Zhang, X. Jia, and C. Liu, "Research on responding ship motion mathematical model," Journal of Dalian Maritime University, vol. 30, no. 1, pp. 18-21, February 2004.

[14] J. Yu and Z. Shao, "Kinetic simulating study on the model of the ship maneuvering motion," Journal of Jimei University (Natural Science), vol. 7, no. 3, pp. 249-253, September 2002.

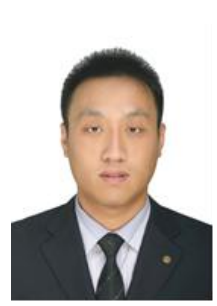

Yanfei Tian was born in 1983 and Now He is a Ph.D candidate in School of Navigation, Wuhan University of Technology (WUT), majoring in transportation information engineering and control and devoting himself to intelligent navigation and simulation technology, marine risk evaluation and safety guard. He received his master's degree from WUT in 2013. Tian had been also crew member on board ship and he is a 2nd bridge officer now.

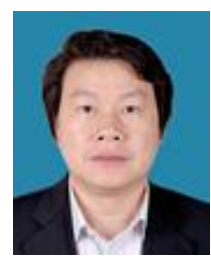

Liwen Huang was born in 1965 and he is a professor and a doctorial supervisor in School of Navigation, Wuhan University of Technology (WUT), devoting himself to intelligent navigation and simulation technology, maritime safety guard, etc., He received his doctor's degree from Qingdao Ocean University (now renamed Ocean University of China) in 1997. Prof. Huang had been a postdoctor in the Ship and Ocean Engineering Post-doctoral Research Station located in Wuhan Transportation University (WTU, which is one of the predecessors of the current WUT) from 1997.9 to 2000.7, and in the Institute of Atmospheric Physics, Chinese Academy of Sciences.

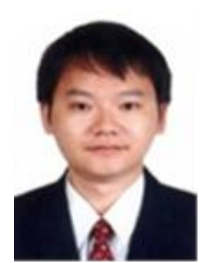

Yong Xiong was born in 1976 and he is an associate professor and a master's supervisor in School of Navigation, Wuhan University of Technology (WUT), devoting himself to control theory, control engineering and ship intelligent control technology, received his doctor's degree from Zhejiang University in 2005. Prof Xiong had been a postdoctor in the Computer Application Post-doctoral Research Station located in South China University of Technology from 2006 to 